Chirurg 2012 $\cdot 83: 386$

DOI 10.1007/s00104-012-2290-1

Online publiziert: 16. März 2012

(c) Springer-Verlag 2012

O. Strobel · M.W. Büchler

Klinik für Allgemein-, Viszeral- und Transplantationschirurgie, Universität Heidelberg

\title{
Roboterassistierte vs. offene Pankreatoduodenektomie
}

\section{Zu früh für eine valide Vergleichsstudie}

\section{Originalpublikation}

Buchs NC, Addeo P, Bianco FM et al (2011)

Robotic versus open pancreaticoduodenec-

tomy: a comparative study at a single institu-

tion. World J Surg 35:2739-2746

\section{Hintergrund und Fragestellung}

Obwohl die laparoskopische Pankreatoduodenektomie bereits 1994 erstmals beschrieben wurde, wird sie wegen der aufwendigen Resektion und insbesondere wegen der komplexen Rekonstruktion bis heute nur in wenigen Zentren regelmäßig durchgeführt. Die Nachteile im Vergleich zur offenen Operation werden vor allem in einer verlängerten Operationszeit und Einschränkungen bezüglich der onkologischen Radikalität und der sicheren Anastomosierung insbesondere des Pankreas gesehen.

Buchs und Kollegen publizierten nun eine vergleichende Studie zum Thema: roboterassistierte vs. offene Pankreatoduodenektomie.

\section{Methoden}

In einer retrospektiven Analyse wurden 44 roboterassistierte (da Vinci) mit 39 offenen Pankreatoduodenektomien verglichen. Die offenen Operationen wurden zwischen 2002 und 2007 von mehreren Chirurgen, die roboterassistierten Operationen zwischen 2007 und 2010 alle von einem einzigen Chirurgen durchgeführt. Es wurden intraoperative Parameter und die perioperativen Ergebnisse verglichen.

\section{Ergebnisse}

Es bestanden demographische Unterschieden zwischen den beiden Gruppen, die Indikationen waren jedoch vergleichbar (75 vs. 69\% Malignome). Als wichtigste Ergebnisse berichten die Autoren eine signifikant verkürzte Operationszeit (444 vs. $559 \mathrm{~min}$ ), einen signifikant reduzierten Blutverlust ( 387 vs. $827 \mathrm{ml}$ ) und eine höhere Anzahl resezierter Lymphknoten $(16,8$ vs. 11$)$ in der roboterassistierten im Vergleich zur offenen Gruppe. Komplikationsraten, Mortalität und Liegedauer waren vergleichbar.

Wichtig für die Interpretation der Daten sind gravierende Unterschiede zwischen den Gruppen bezüglich der Versorgung des Restpankreas: Während in der offenen Gruppe in 95\% eine Pankreatikojejunostomie angelegt wurde und bei nur $5 \%$ eine Sklerosierung des Restpankreas erfolgte, wurde in der roboterassistierten Gruppe in 9\% eine Pankreatikojejunostomie und in 36\% eine Pankreatikogastrostomie angelegt. Bei 55\% der roboterassistiert operierten Patienten wurde eine Pankreasanastomose durch Sklerosierung des Restpankreas umgangen.

\section{Diskussion}

Die Autoren folgern, es sei zwar zu früh für eine definitive Konklusion hinsichtlich der Langzeitergebnisse, leiten aber aus ihren perioperativen Daten einen Trend zugunsten der roboterassistierten Operation ab.

Mehrere Punkte zeigen allerdings, dass die vorliegende Studie diese Schlussfolgerung nicht erlaubt:

- Fehlende Vergleichbarkeit: Die offene Gruppe ist eine historische Vergleichsgruppe, die in einem früheren Zeitraum von anderen Chirurgen, mit geringerer Fallzahl, operiert wurde. Aufgrund der oben genannten, gra- vierenden Unterschiede in der Versorgung des Restpankreas tritt beim Vergleich der beiden Operationsarten der Zugangsweg in den Hintergrund. - Qualität der Kontrollgruppe: Zur Beurteilung jedes neuen Verfahrens ist der Vergleich der Ergebnisse mit dem etablierten Standardverfahren notwendig. Um dies zu ermöglichen, muss die Kontrollgruppe jeder vergleichenden Studie den aktuell gültigen Qualitätsstandards entsprechen. Im konkreten Fall ist zu bezweifeln, dass die offene Gruppe mit einer Operationszeit von über $9 \mathrm{~h}$ und einer mittleren Anzahl von 11 entfernten Lymphknoten diesen Anforderungen gerecht wird.

\section{Fazit}

Buchs et al. zeigen, dass eine StandardPankreatoduodenektomie mit Ausnahme der Pankreasanastomose roboterassistiert sicher durchführbar ist. Für einen Vergleich zur offenen Operation ist es allerdings zu früh. Hierzu sind prospektive Studien mit geeigneten Kontrollgruppen notwendig.

\section{Korrespondenzadresse}

PD Dr. O. Strobel

Klinik für Allgemein-, Viszeral- und Transplantationschirurgie, Universität Heidelberg,

Im Neuenheimer Feld 110, 69120 Heidelberg Oliver.Strobel@med.uni-heidelberg.de

Interessenkonflikt. Der korrespondierende Autor gibt für sich und seinen Koautor an, dass kein Interessenkonflikt besteht. 